\title{
Diffusion-based model to predict the conservation of gratings recorded in poly(vinyl alcohol)-acrylamide photopolymer
}

\author{
Sergi Gallego, Cristian Neipp, Manuel Ortuño, Andrés Márquez, Augusto Beléndez, \\ and Inmaculada Pascual
}

\begin{abstract}
Photopolymers are interesting materials for use in recording information in holography. We study the holographic behavior and stability of volume holograms recorded in poly(vinyl alcohol)-acrylamide photopolymers with and without a cross linker. Using a first-harmonic diffusion model, we analyze the residual monomer that remains when volume diffraction gratings are recorded in photopolymer materials. The importance of this residual monomer to the stability of the gratings is evaluated. (C) 2003 Optical Society of America

OCIS codes: $\quad 090.0090,090.2900,090.7380$.
\end{abstract}

\section{Introduction}

There has been much research to characterize polymers as holographic recording materials because polymers have many attractive features. ${ }^{1}$ We could mention their ability to self-develop, the fact that dry processing can be used with them, their good stability and thick emulsion layers, and their high sensitivity, diffraction efficiency, and resolution.

Photopolymer systems for recording holograms typically comprise one or more monomers, a photoinitiation system, and an inactive component that is often referred to as a binder. Other components are sometimes added to control a variety of properties such as sensitivity and viscosity of the recording medium. To obtain high-quality volume holograms in photopolymers, one must optimize the composition of these materials. To do this it is important to understand the mechanism of hologram formation in photopolymer materials. Although the mechanism is complex, it is well established that two processes, i.e., monomer polymerization and monomer diffusion, are

S. Gallego, M. Ortuño, and I. Pascual (e-mail: pascual@ua.es) are with the Departamento Interuniversitario de Óptica, Universidad de Alicante, Apartado 99, E-03080 Alicante, Spain. C. Neipp, A. Márquez, and A. Beléndez are with the Departamento de Física, Ingeniería de Sistemas y Teoría de la Señal, Universidad de Alicante, Apartado 99, E-03080 Alicante, Spain.

Received 14 March 2003; revised manuscript received 16 July 2003.

0003-6935/03/295839-07\$15.00/0

(C) 2003 Optical Society of America chiefly responsible for gratings formation. Several theoretical models have been proposed in which these processes are taken into account; all of them are highly predictive. ${ }^{2-7}$ In particular, the firstharmonic model suggested by Piazolla and Jenkins ${ }^{5}$ has the advantage that a closed-form expression of the refractive-index modulation was obtained, which makes the model easy to handle. We have proposed another first-harmonic diffusion model, ${ }^{7}$ in which the differences between the refractive indices of the monomer and the polymer are also considered. One of the features of our model is that it predicts that there will still be a free monomer remaining in the material after the gratings are formed, even for long exposure times. As we discuss below, this residual monomer influences the conservation of the gratings stored in the polymer. Therefore it is useful to predict accurately the amount of the monomer that will remain in the material after exposure.

Our experiments were carried out with a poly(vinyl alcohol)-acrylamide photopolymer. The behavior of this photopolymer during recording is well known, ${ }^{8-13}$ but the behavior after this stage has not been studied in depth. In general, especially with acrylic photopolymers, ultraviolet light is used to stabilize the photopolymer after the holographic grating is recorded. ${ }^{14}$ This is done because there is a component that is photosensitive in the ultraviolet band that consumes the dye. In our case, we cannot cure the photopolymer because it does not contain this component. We therefore make use of other methods to stabilize the recorded grating: We introduce a cross linker to con- 
Table 1. Components of the Photopolymerizable Solution without a Cross Linker (Type 1)

\begin{tabular}{cc}
\hline Component & Value \\
\hline AA & $0.44 \mathrm{M}$ \\
TEA & $0.20 \mathrm{M}$ \\
YE & $2.5 \times 10^{-4} \mathrm{M}$ \\
PVA & $7 \%$ weight/volume \\
\hline
\end{tabular}

serve the grating in the dark, and we expose the grating to incoherent white light. In this paper we study the conservation of volume holograms recorded in two types of photopolymer: one in which only one monomer, acrylamide (AA), is used and another in which a cross linker, $N, N^{\prime}$-methylenebisacrylamide (BMAA), is added. We compare our experimental results with the theoretical results that we obtained by using our new diffusion model.?

\section{Experimental Setup}

A photopolymerizable solution was prepared in case under red light by the addition to poly(vinyl alcohol) (PVA) of yellowish eosin (YE) together with a triethanolamine solution. The concentration of each of the components dissolved in water is listed in Table 1 for type 1 photopolymer (without a cross linker) and in Table 2 for type 2 photopolymer (with a cross linker). However, the concentration of BMAA is limited to $0.05 \mathrm{M}$ because BMAA does not dissolve well in water. The resultant solution was deposited onto a $20 \mathrm{~cm} \times 40 \mathrm{~cm}$ glass plate by an automatic depositor, and the thickness of the film was adjusted. The plate was dried for a period of $48 \mathrm{~h}$ in the dark and under normal laboratory conditions (temperature, $21-23{ }^{\circ} \mathrm{C}$; relative humidity $40-60 \%$ ). Once dried, it was cut into plates that measured $6.5 \mathrm{~cm} \times 6.5 \mathrm{~cm}$ to be used in our experimental setup, and the thickness, absorption constant, and refractive-index modulation were measured by optical methods. ${ }^{15,16}$

To study the behavior of the photopolymer as a holographic recording material we obtained diffraction gratings by using a holographic setup. The experimental device is shown in Fig. 1. An argon laser at a wavelength of $514 \mathrm{~nm}$ was used to store diffraction gratings by means of continuous laser exposure. The laser beam was split into two secondary beams with an intensity ratio of $1: 1$. The diameter of these beams was increased to $1 \mathrm{~cm}$ with an expander, while spatial filtering was ensured. The object and reference beams were recombined at the sample at an

Table 2. Components of the Photopolymerizable Solution with a Cross Linker (Type 2)

\begin{tabular}{cc}
\hline Component & Value \\
\hline AA & $0.40 \mathrm{M}$ \\
BMAA & $0.04 \mathrm{M}$ \\
TEA & $0.20 \mathrm{M}$ \\
YE & $2.5 \times 10^{-4} \mathrm{M}$ \\
PVA & $7 \%$ weight $/$ volume \\
\hline
\end{tabular}

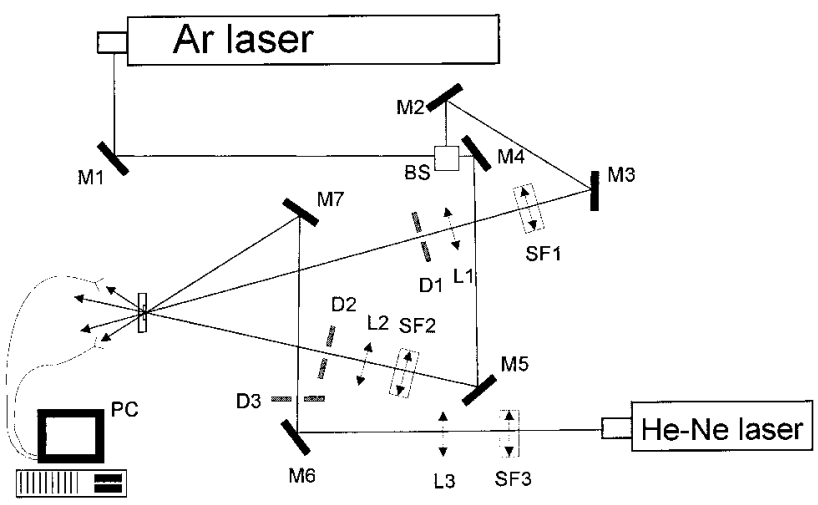

Fig. 1. Experimental setup: BS, beam splitter; $\mathrm{M}_{i}$, mirrors; $\mathrm{SF}_{i}$, spatial filters; $\mathrm{L}_{i}$, lenses; $\mathrm{D}_{i}$, diaphragms; PC, personal computer.

angle of $16.8^{\circ}$ to the normal with an appropriate set of mirrors, and the spatial frequency obtained was 1125 lines $/ \mathrm{mm}$. The working intensity at $514 \mathrm{~nm}$ was $6 \mathrm{~mW} / \mathrm{cm}^{2}$. The diffracted and transmitted intensities were monitored in real time with a $\mathrm{He}-\mathrm{Ne}$ laser positioned at Bragg's angle $\left(20.8^{\circ}\right)$ tuned to 633 $\mathrm{nm}$, where the material does not polymerize. To obtain the transmittance and diffraction efficiencies as a function of the angle at reconstruction we placed the plates on a rotating stage. We calculated transmittance and diffraction as the ratio of transmitted and diffracted beams, respectively, to the incident power, and to take Fresnel losses into account we multiplied the expression by an appropriate factor.

\section{Theoretical Model}

In Ref. 7 a first-harmonic diffusion model was proposed to explain the mechanism of hologram formation in photopolymers. This model is based on the following two equations, which describe the decrease in free monomers and the creation of polymers as a function of time when the material is illuminated:

$$
\begin{aligned}
& \frac{\partial \phi^{(m)}}{\partial t}=-k_{R}(t) I(x) \phi^{(m)}(x, t)+\frac{\partial}{\partial x} D \frac{\partial}{\partial x} \phi^{(m)}(x, t), \\
& \frac{\partial \phi^{(p)}}{\partial t}=k_{R}(t) I(x) \phi^{(m)}(x, t),
\end{aligned}
$$

where $\phi$ are the volume fractions of the various compounds; $(m)$ and $(p)$ stand for monomer and polymer, respectively; $D$ is the diffusion constant, which we assume to be constant; $I$ is the illumination intensity; and $k_{R}(t)$ is the polymerization rate, which we assume decays exponentially with time in the following way:

$$
k_{R}(t)=k_{0} \exp (-\varphi t) .
$$

If the polymeric material is exposed to an interference pattern of the form

$$
I(x)=I_{0}\left\lfloor 1+m \cos \left(K_{g} x\right)\right\rfloor,
$$


where $m$ is the beam intensity modulation $(m=1$ in our calculations), $K_{g}$ is the grating wave number, and $I_{0}$ is the average recording intensity, the monomer concentration can be expressed as

$$
\phi^{(m)}(x, t)=\phi_{0}^{(m)}(t)-\phi_{1}^{(m)}(t) \cos \left(K_{g} x\right) .
$$

Equation (5) explains the fact that the consumption of monomer is more rapid in bright regions than in dark ones. ${ }^{5}$ In the bright regions the monomer is converted into polymer, so the polymer concentration can be expressed as

$$
\phi^{(p)}(x, t)=\phi_{0}^{(p)}(t)+\phi_{1}^{(p)}(t) \cos \left(K_{g} x\right) .
$$

By substituting Eqs. (4)-(6) into Eqs. (1) and (2) we obtain

$$
\begin{aligned}
\frac{\partial}{\partial t} \phi^{(m)}(x, t)= & -k_{R}(t) I_{0}\left[1+m \cos \left(K_{g} x\right)\right]\left[\phi_{0}^{(m)}\right. \\
& \left.-\phi_{1}^{(m)} \cos \left(K_{g} x\right)\right]+\frac{\phi_{1}^{(m)}}{\tau_{D}} \cos \left(K_{g} x\right), \\
\frac{\partial}{\partial t} \phi^{(p)}(x, t)= & k_{R}(t) I_{0}\left[1+m \cos \left(K_{g} x\right)\right]\left[\phi_{0}^{(m)}\right. \\
& \left.-\phi_{1}^{(m)} \cos \left(K_{g} x\right)\right] .
\end{aligned}
$$

The diffusion time, $\tau_{D}$, is defined as $\tau_{D}=1 / D K_{g}{ }^{2}$.

Following a treatment similar to that of Aubrecht et al. ${ }^{3}$ the first-harmonic component of the refractive index can be related to the first-harmonic components of the polymer and monomer concentrations as

$$
\begin{aligned}
n_{1}= & \frac{\left(n_{\text {dark }}{ }^{2}+2\right)^{2}}{3 n_{\text {dark }}}\left[-\left(\frac{n_{m}{ }^{2}-1}{n_{m}{ }^{2}+2}-\frac{n_{b}{ }^{2}-1}{n_{b}{ }^{2}+2}\right) \phi_{1}{ }^{(m)}\right. \\
& \left.+\left(\frac{n_{p}{ }^{2}-1}{n_{p}{ }^{2}+2}-\frac{n_{b}{ }^{2}-1}{n_{b}{ }^{2}+2}\right) \phi_{1}{ }^{(p)}\right]
\end{aligned}
$$

where $n_{\text {dark }}$ is the refractive index of the mixture of compounds without illumination and $n_{p}, n_{m}$, and $n_{b}$ are the refractive indices of the polymer, the monomer, and the binder, respectively.

Finally, from Eqs. (5)-(8) and after some calculations the following expressions for the harmonic terms can be derived:

$$
\begin{aligned}
& \frac{\mathrm{d} \phi_{0}^{(m)}}{\mathrm{d} t}=-k_{R}(t) I_{0}\left[\phi_{0}^{(m)}-\frac{1}{2} \phi_{1}^{(m)}\right], \\
& \frac{\mathrm{d} \phi_{1}^{(m)}}{\mathrm{d} t}=k_{R}(t) I_{0}\left[\phi_{0}^{(m)}-\phi_{1}^{(m)}\right]-\frac{\phi_{1}^{(m)}}{\tau_{D}}, \\
& \frac{\mathrm{d} \phi_{0}^{(p)}}{\mathrm{d} t}=k_{R}(t) I_{0}\left[\phi_{0}^{(m)}-\frac{1}{2} \phi_{1}^{(m)}\right], \\
& \frac{\mathrm{d} \phi_{1}^{(p)}}{\mathrm{d} t}=k_{R}(t) I_{0}\left[\phi_{0}^{(m)}-\phi_{1}^{(m)}\right] .
\end{aligned}
$$

Equations (10)-(13) combined with Eqs. (3) and (9) are the basic equations of this first-harmonic diffu-

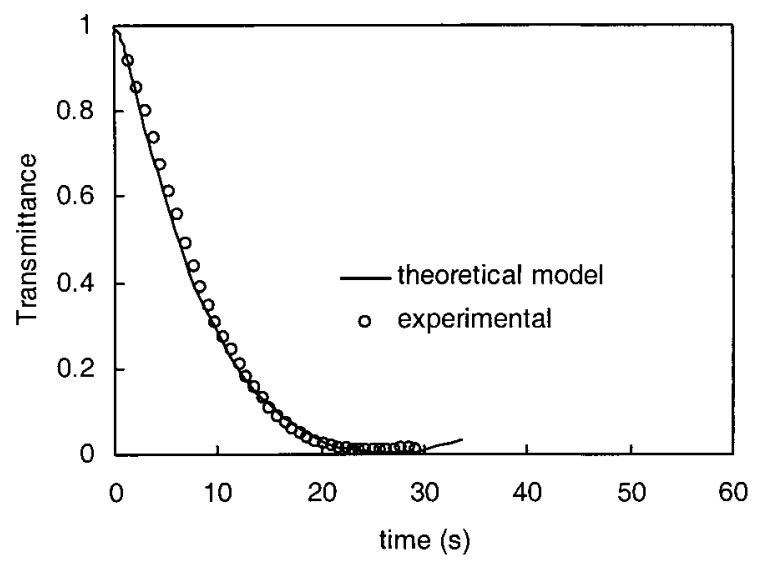

Fig. 2. Transmission efficiency versus time for an $87-\mu \mathrm{m}$-thick type 1 photopolymer during holographic recording.

sion model. If we fit this model to the behavior exhibited by our PVA-AA-based photopolymer when the grating is being stored (as we discuss in Section 4 below), two findings may be mentioned: high values of diffusion time, $\tau_{D}$, in all cases near $30 \mathrm{~s}$, and values of $\varphi$ above 0.0015 . These values of $\tau_{D}$ and $\varphi$ mean that our model predicts the existence of a significant amount of residual monomer in the dark regions, which, because diffusion is slow (high values of $\tau_{D}$ ) and the polymerization rate decreases rapidly, does not move to the bright regions where there is a lower monomer concentration.

\section{Results and Discussion}

Figure 2 shows transmission efficiency versus time for a diffraction grating recorded on photopolymer layers in which AA is the only monomer (material type 1). Open circles correspond to the experimental data, whereas the continuous curve corresponds to the theoretical fit with the first-harmonic diffusion. For the theoretical curve the following values were used: diffusion time $\left(\tau_{D}\right), 30 \mathrm{~s} ;(\varphi)=0.0015 \mathrm{~s}^{-1}$; initial polymerization rate $\left(k_{0}\right), 0.02 \mathrm{~cm}^{2} \mathrm{~mW}^{-1} \mathrm{~s}^{-1}$; incident intensity, $6 \mathrm{~mW} / \mathrm{cm}^{2}$; refractive index of the monomer $\left(n_{m}\right), \sim 1.56$; refractive index of polymer $\left(n_{p}\right), 1.60$; refractive index of binder $\left(n_{b}\right), 1.51$; refractive index of the dry photopolymer film before being exposed $\left(n_{\text {dark }}\right), 1.52$; and thickness $87 \mu \mathrm{m}$, which we obtained by using a theoretical fit (based on Kogelnik's theory for pure phase, unslanted transmission gratings $13,15,16$ of the angular response obtained after recording. From this figure it can be seen that the experimental data may be modeled based on the fitting performed with the model used, and there is good agreement between the theoretical and experimental data. Furthermore, we obtain a transmission efficiency (TE) of less than 5\%, which means a diffraction efficiency of more than $95 \%$ for an exposure energy of $180 \mathrm{~mJ} / \mathrm{cm}^{2}$ because the intensity of the source is $6 \mathrm{~mW} / \mathrm{cm}^{2}$.

Figure 3 shows the theoretical values, predicted by the model proposed, of the average monomer concentration that remains on the plate divided by the ini- 


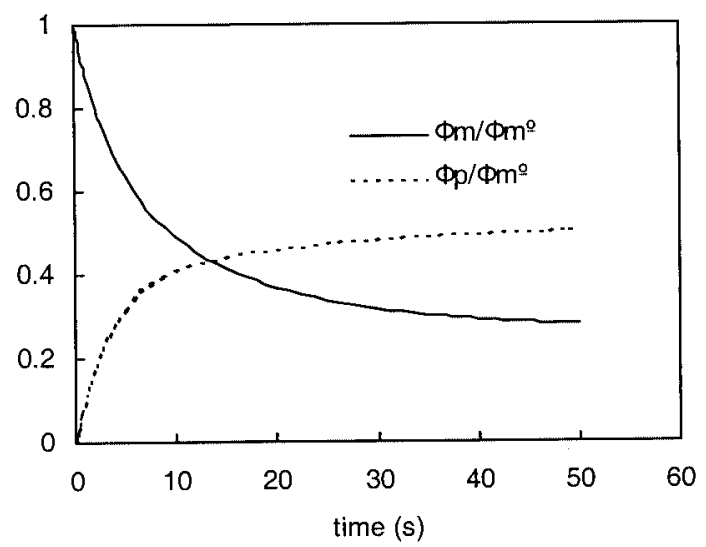

Fig. 3. Monomer concentration $(\Phi m)$ divided by initial monomer concentration $\left(\Phi m^{\circ}\right)$ and polymer concentration $(\Phi p)$ divided by initial monomer concentration as a function of time for an $87-\mu \mathrm{m}-$ thick type 1 photopolymer plate during holographic recording.

tial monomer concentration and of the average polymer concentration generated as a function of time also divided by the initial monomer concentration. As can be seen from Fig. 2, the theoretical TE is minimum for a time $(t)$ of $30 \mathrm{~s}$. For the same period of time as shown in Fig. 3, we can determine the amounts of polymer and residual monomer present in the material. As can be seen, there is an amount of residual monomer that could polymerize if the plate were exposed to light. Figure 4 shows transmission efficiency as a function of the angle at reconstruction, where this angular response was measured after $30 \mathrm{~s}$. However, this optimum angular response that may be observed in Fig. 4 is not conserved with time, even when the stored grating is kept in the dark. This fact is illustrated by Fig. 5, which shows the fraction of refractive-index modulation with respect to initial modulation as a function of the time elapsed after the grating was recorded. As can be seen, the index modulation decreases initially until it reaches a certain value and then stabilizes. A minimum of $20 \%$ of the index modulation obtained during recording is lost.

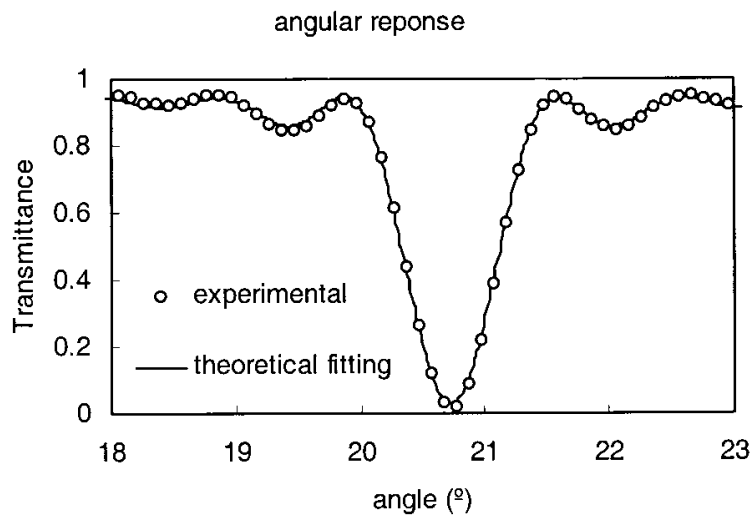

Fig. 4. TE as a function of the angle at reconstruction immediately after exposure for an $87-\mu \mathrm{m}$-thick type 1 photopolymer $(\Delta n=$ 0.0030).

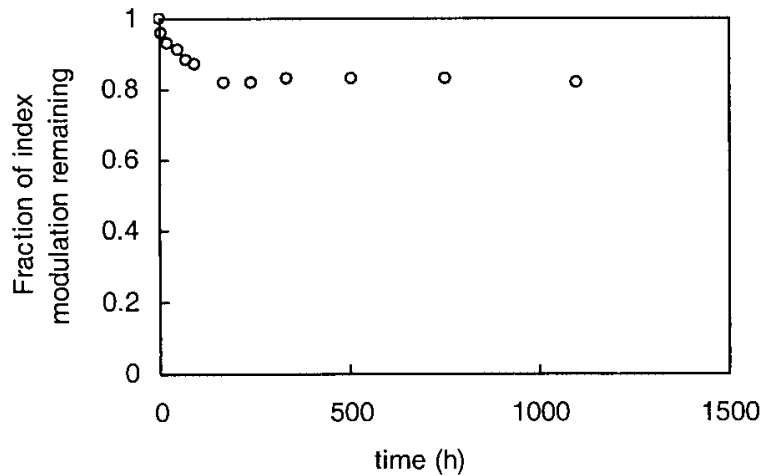

Fig. 5. Refractive-index modulation divided by the index modulation obtained immediately after recording as a function of time when the grating is kept in the dark.

If immediately after the grating is recorded, however, it is directly exposed to the incoherent light of a fluorescent tube with an illuminance $E=600$ lux, the index modulation decreases by almost 30\% (Fig. 6). This decrease in index modulation is produced by the polymerization of the residual monomer. As there is a quantity of triethanolamine (TEA) and YE in nonexposed zones, the monomer that is present in greater amounts in the nonexposed zones polymerizes when the plate is exposed to ambient light, thereby making the difference in refractive index between the illuminated and nonilluminated zones decrease. It is obvious that most of the residual monomer is found in the nonexposed zones, because from Figs. 2 and 3 we can see that a point is reached at which, however much the energy with which we illuminate the plate is increased, the grating hardly changes and the amount of monomer neither changes nor decreases substantially. In other words, almost no monomer appears to remain in the bright regions.

When we compare the behavior of the gratings stored in the dark (Fig. 5) with those stored in the light (Fig. 6), we might think that the phenomena that cause the loss of index modulation are analogous. However, if the grating that was kept in the dark once its index modulation had stabilized is illu-

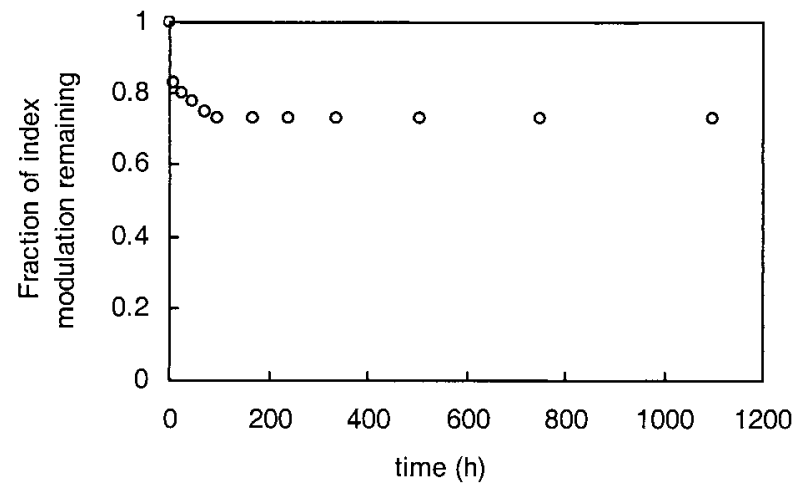

Fig. 6. Refractive-index modulation divided by the index modulation obtained immediately after recording as a function of time when the grating is kept in the light. 


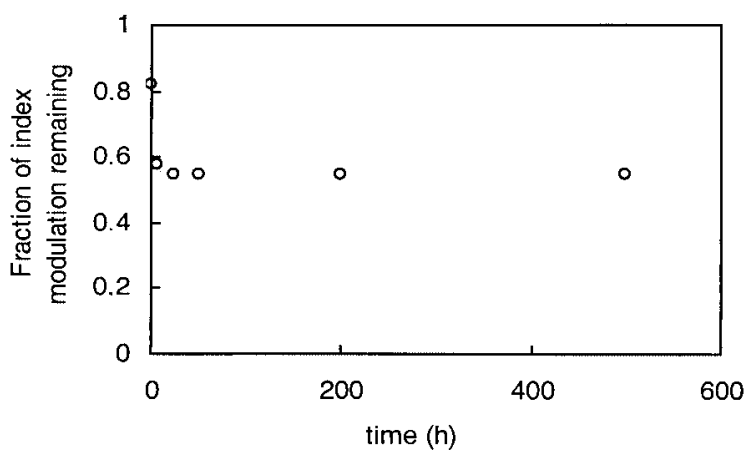

Fig. 7. Refractive-index modulation divided by the index modulation obtained immediately after recording as a function of time when the grating is exposed to light after $1100 \mathrm{~h}$ in the dark.

minated after 1100 hours (rightmost point in Fig. 5), we can see that it again loses almost $30 \%$ of the index modulation reached after recording (Fig. 7). From Fig. 7 it can be deduced that this loss occurs within $\sim 30 \mathrm{~h}$, and that from then on the index modulation remains constant, even after more than $1000 \mathrm{~h}$. A possible reason for this loss of index modulation in the dark could be the diffusion that takes place in the material, as was pointed out in other studies. 8,13

To prevent this loss of index modulation in the dark we included in the composition a cross-linker BMAA, which is a bifunctional monomer (type 2). This enabled us to obtain chains of polyacrylamide linked together by BMAA. Therefore, with the same total initial monomer concentration, we achieved much higher index modulations. After the theoretical fits the values of the parameters our model were obtained. Now $n_{p}$ was a little greater, so we took $n_{p}=$ 1.63. Constant $k_{0}$ also increased, as was to be expected, because adding a cross-linker monomer favors the polymerization mechanism, so $k_{0}$ now took the value $0.024 \mathrm{~cm}^{2} \mathrm{~mW}^{-1} \mathrm{~s}^{-1}$. All these changes resulted in an improvement in the energetic sensitivities (of less than $80 \mathrm{~mJ} / \mathrm{cm}^{2}$ ) even for slightly thinner plates, as can be seen from Fig. 8, which shows $\mathrm{TE}$ as a function of time for a $77-\mu \mathrm{m}$-thick plate. From this figure we can see that in only $10 \mathrm{~s}$ a minimum TE is obtained, and this implies a maximum diffraction efficiency. Nevertheless, there is also a significant amount of residual monomer, as can be seen from Fig. 9, in which after $10 \mathrm{~s}$ there still remains approximately $45 \%$ free monomer. If we had not stopped recording after $10 \mathrm{~s}$, the monomer would have continued to be consumed, giving rise to overmodulation effects that have already been analyzed in other studies. . $^{13,16}$

When the grating is kept in the dark, we see that there are no modifications in the angular response, even after 2000 h. In Fig. 10, which shows the angular response of the grating stored in the photopolymer with AA and BMAA, theoretical fits are based on Kogelnik's theory, and the parameters obtained are the same in a period of $2000 \mathrm{~h}$. This shows that with this composition it is possible to conserve diffraction gratings with an optimum angular response to be

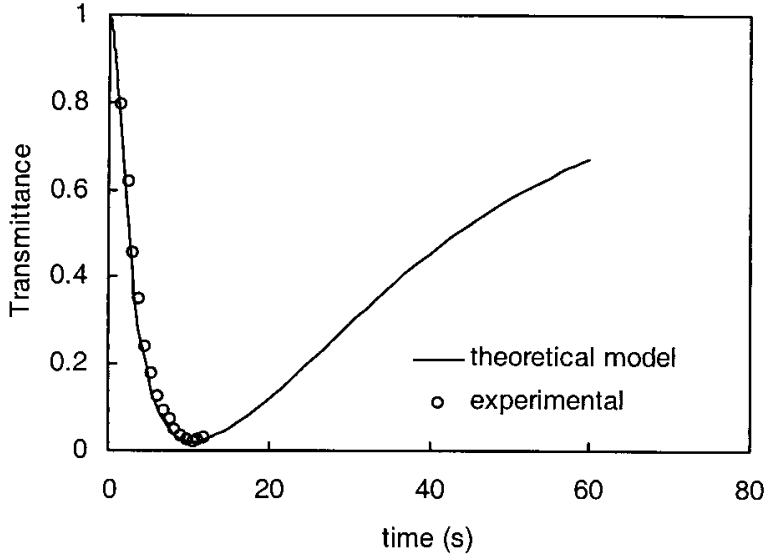

Fig. 8. Transmission efficiency versus time for a $77-\mu \mathrm{m}$-thick type 2 photopolymer.

read with red light, to which our dye is not sensitive. However, because of the residual monomer, when the grating is exposed to the light after recording there continues to be a loss of index modulation. This loss

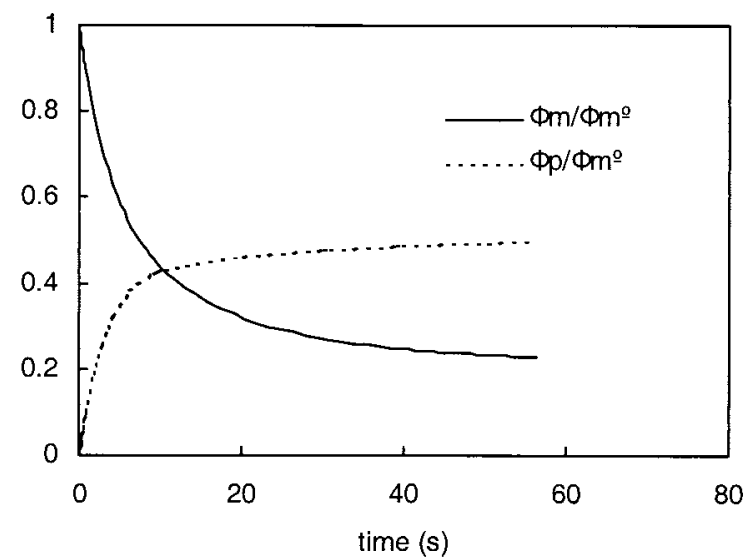

Fig. 9. Monomer concentration ( $\Phi m)$ divided by initial monomer concentration $\left(\Phi m^{\circ}\right)$ and polymer concentration $(\Phi p)$ divided by initial monomer concentration as a function of time for a $77-\mu \mathrm{m}$ thick type 2 photopolymer plate.

angular reponse

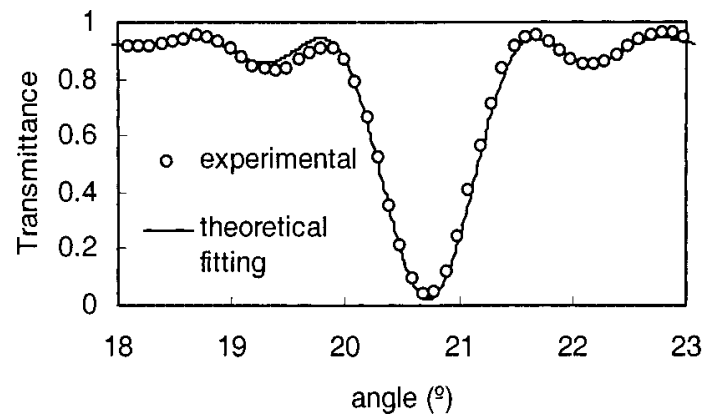

Fig. 10. TE as a function of angle at reconstruction immediately after exposure for a 77- $\mu$ m-thick type 2 photopolymer $(\Delta n=$ $0.0035)$. 


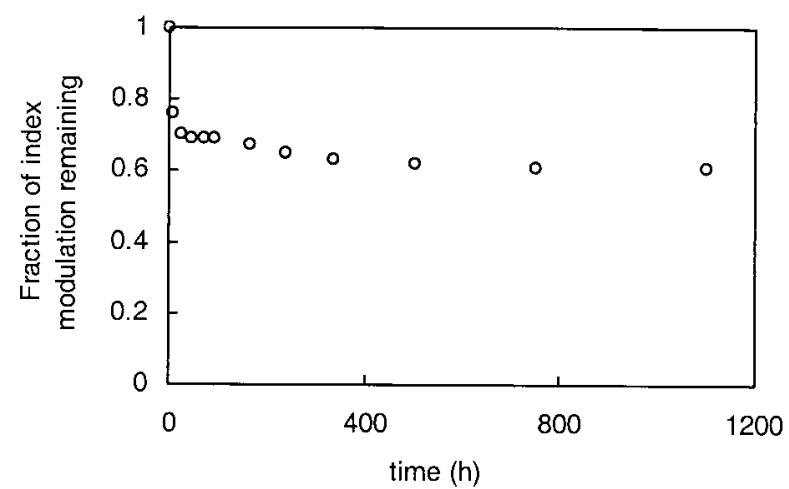

Fig. 11. Refractive-index modulation divided by the index modulation obtained immediately after recording as a function of time when the grating is kept in the light.

occurs because polymerization takes place in both the exposed and the unexposed zones because of the uniform illumination with white light, as there is monomer present in both zones. For this reason the loss of modulation is not proportional to the amount of residual monomer. Therefore we lose only $40 \%$ of the initial index modulation, as can be seen from Fig. 11, which shows the index modulation that is left compared with that which existed initially.

To reduce the amount of monomer in the gratings recorded in the photopolymer with BMAA we must prepare thinner plates. In this case, with thinner layers a higher index modulation must be obtained for a minimum transmission efficiency to be achieved, so more monomer is consumed. Because there is less residual monomer in these cases, when the grating is exposed to the light the decrease in index is significantly less. These assumptions were confirmed when we studied a $53-\mu \mathrm{m}$ plate with BMAA. From Fig. 12 it can be seen that for exposures of $40 \mathrm{~s}$ there is no overmodulation of the refractive index; instead, our grating exhibits a minimum TE and, if exposure is continued, this situation hardly varies because almost all the monomer must be located in the unexposed zones. Furthermore, as can be seen from Fig. 13, in our model after $40 \mathrm{~s}$, less than $25 \%$ of

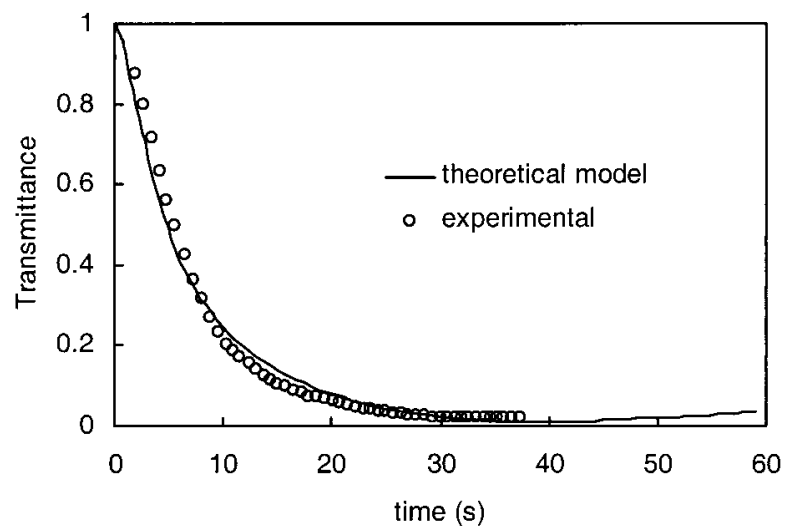

Fig. 12. Transmission efficiency versus time for a $53-\mu \mathrm{m}$-thick type 2 photopolymer.

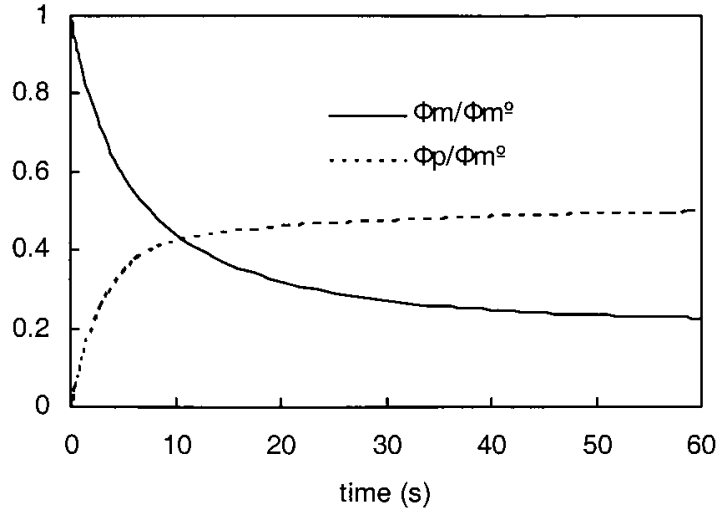

Fig. 13. Monomer concentration $(\Phi m)$ divided by initial monomer concentration $\left(\Phi m^{\circ}\right)$ and polymer concentration $(\Phi p)$ divided by initial monomer concentration as a function of time for a $53-\mu \mathrm{m}-$ thick type 2 photopolymer plate.

the initial monomer is not polymerized. If we look at Fig. 14, which shows conservation of index modulation as a function of time, we can see that once our grating is stabilized, only $20 \%$ of the initial index modulation is lost.

\section{Conclusions}

We have obtained holographic diffraction gratings by using two different compositions of poly(vinyl alcohol)-acrylamide photopolymer: one without a cross linker (type 1) and the other with a cross linker (type 2).

We made a thorough study of the conservation of the holograms recorded in photopolymers of the two compositions. With each composition we studied conservation of the hologram in the dark (with red light) and when it was exposed to white light.

When we modeled the behavior of the photopolymer during the recording stage, the importance of the residual monomer in the conservation of the gratings was seen, together with the accuracy of the amount of monomer predicted.

A $20 \%$ loss of index modulation was seen when the grating was stored in type 2 composition PVA-AA in

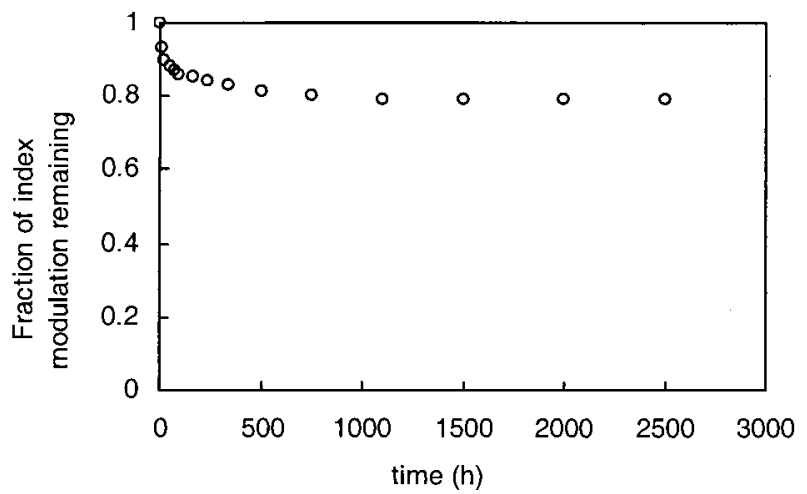

Fig. 14. Refractive-index modulation divided by the index modulation obtained immediately after recording as a function of time when the grating is kept in the light. 
the dark, and an additional 30\% of index modulation was lost when the plate was subsequently exposed to light, which means a total loss of $50 \%$ of index modulation. It was also shown that the mechanisms by which the index modulation decreases in the dark and subsequently when the plate is exposed to the light are different.

When the cross-linker BMAA is added (type 2 composition) it is possible to obtain holograms that are stable with time if they are read only with red light, that is, if they are kept in the dark. In addition, if we choose the correct thickness for the photopolymer plate, only $20 \%$ of index modulation will be lost if the plate is exposed to the light.

This research was supported by the Ministerio de Ciencia y Tecnología, Comisión Interministerial de Ciencia y Tecnología, Spain, under project MAT20001361-C04-04 and by the Oficina de Ciencia y Tecnología (Generalitat Valenciana, Spain) under project GV01-130.

\section{References}

1. R. A. Lessard and G. Manivannan, eds., Selected Papers on Photopolymers, Vol. 114 of SPIE Milestone Series (SPIE Optical Engineering Press, Bellingham, Wash., 1996).

2. G. Zhao and P. Mourolis, "Diffusion model of hologram formation in dry photopolymers materials," J. Mod. Opt. 41, 19291939 (1994).

3. I. Aubrecht, M. Miler, and I. Koudela, "Recording of holographic diffraction gratings in photopolymers: theoretical modelling and real-time monitoring of grating growth," J. Mod. Opt. 45, 1465-1477 (1998).

4. J. H. Kwon, H. C. Hwang, and K. C. Woo, "Analysis of temporal behavior of beams diffracted by volume gratings formed in photopolymers," J. Opt. Soc. Am. B 16, 1651-1657 (1999).

5. S. Piazolla and B. J. Jenkins, "First-harmonic diffusion model for holographic grating formation in photopolymers," J. Opt. Soc. Am. B 17, 1147-1157 (2000).
6. J. T. Sheridan, J. R. Lawrence, and F. T. O'Neill, "Diffusion based model of holographic grating formation in photopolymers: generalized non-local material responses," J. Opt. A 3, 477-488 (2001)

7. C. Neipp, S. Gallego, M. Ortuño, A. Márquez, M. Álvarez, A. Beléndez, and I. Pascual, "First-harmonic diffusion-based model applied to a polyvinyl-alcohol-acrylamide-based photopolymer," J. Opt. Soc. Am. B 20, 2052-2060 (2003).

8. S. Martin, P. E. L. G. Leclere, V. Toal, and Y. F. Lion, "Characterization of an acrylamide-based dry photopolymer holographic recording material," Opt. Eng. 33, 3942-3946 (1994).

9. V. Weiss, E. Millul, and A. Friesem, "Photopolymeric holographic recording media: in situ and real-time characterization," in Holographic Materials II, T. J. Trout, ed. Proc. SPIE 2688, 11-21 (1996).

10. S. Blaya, L. Carretero, R. Mallavia, A. Fimia, M. Ulibarrena, and D. Levy, "Optimization of an acrylamide-based dry film used for holographic recording," Appl. Opt. 37, 7604-7610 (1998).

11. C. García, A. Fimia, and I. Pascual, "Diffraction efficiency and signal-to-noise ratio of diffuse-object holograms in real time in polyvinyl alcohol photopolymers," Appl. Opt. 38, 5548-5551 (1999).

12. C. García, A. Fimia, and I. Pascual, "Holographic behavior of a photopolymer at high thicknesses and high monomer concentrations: mechanism of polymerization," Appl. Phys. B 72, 311-316 (2001).

13. S. Gallego, M. Ortuño, C. Neipp, C. García, A. Beléndez, and I. Pascual, "Temporal evolution of the angular response of a holographic diffraction grating in PVA/acrylamide photopolymer," Opt. Express 11, 181-190 (2003), http://www.opticsexpress.org.

14. J. A. Jenney, "Holographic recording with photopolymers," J. Opt. Soc. Am. 60, 1155-1161 (1970).

15. H. Kogelnik, "Coupled wave theory for thick hologram gratings,” Bell Syst. Tech. J. 48, 2909-2947 (1969).

16. S. Gallego, M. Ortuño, C. Neipp, C. García, A. Beléndez, and I. Pascual, "Overmodulation effects in volume holograms recorded on photopolymers," Opt. Commun. 215, 263-269 (2003). 